\title{
COMPRENDRE \\ Le capteur de distance chromatique, une révolution en marche
}

\author{
Cosimi CORLETO \\ Laurent FLEURY \\ Marie-Agnès DOUGLAS \\ STIL S.A.S., Aix-en-Provence \\ contact@stilsa.com
}

\begin{abstract}
Il existe une très grande variété de technologies de mesure de distance sans contact, dans le domaine optique, la plus connue et la plus diffusée d'entre elles étant le capteur à triangulation laser. Néanmoins le laser atteint ses limites en capacité de détection alors que la plupart des secteurs d'activité produisent des pièces de plus en plus petites (miniaturisation) et de plus en plus complexes (multicouche, impression 3D, composites, nanomatériaux...).
\end{abstract}

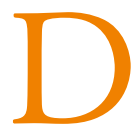

epuis quelques années, une technologie « Made in France » émerge et s'impose progressivement sur la plupart des marchés comme technologie universelle pour les mesures de grande précision. Les possibilités étendues de cette technologie, dite « confocale chromatique », permettent de voir l'apparition d'une nouvelle génération de capteurs qui vont révolutionner la manière de mesurer les distances, de contrôler les dimensions ou encore de générer des images $3 \mathrm{D}$ avec des précisions du micromètre et même submicronique.

Ces capteurs de distance chromatique appartiennent à la famille des capteurs optoélectroniques destinés à mesurer des longueurs. Ils sont donc capables de mesurer de nombreux paramètres : position, distance, dimension, épaisseur, rugosité, vibration, déformation ou encore déplacement. Cette mesure qui était uniquement ponctuelle à l'origine (capteur point), se décline aujourd'hui en version multipoints ce qui permet d'accélérer considérablement l'acquisition des données.

L'origine de la technologie chromatique confocale remonte à 1993 lorsque J. Cohen-Sabban dépose un brevet. Ce brevet apporte alors une innovation décisive par rapport aux travaux de ses prédécesseurs dans le domaine. En effet, c'est en 1957 que Minsky décrit pour la première fois un principe de mesure de distance confocale qui n'est alors que monochromatique. L'introduction du concept chromatique par la société STIL, va révolutionner la technologie confocale classique en substituant un champ chromatique permanent au dispositif d'asservissement mécanique jusqu'alors nécessaire pour permettre l'exploitation du principe confocal. Dès lors la mesure de distance devient à la fois plus précise et plus rapide. Les domaines d'application vont considérablement se développer au cours des 20 dernières années en sortant des laboratoires de recherche pour se déployer progressivement sur l'ensemble des secteurs d'activité industriels.

\section{Mesure sans contact}

Le confocal chromatique appartient à la famille des techniques de mesure sans contact. De manière générale, cette famille inclut les capteurs optiques mais aussi d'autres technologies telles que les capteurs à ultrasons, capacitifs ou encore inductifs.

Les capteurs ultrasons génèrent un bruit de fréquence(s) connue(s) par la mise sous tension d'un matériau piézo-électrique. L'écho de retour de la surface agit sur le piézo qui

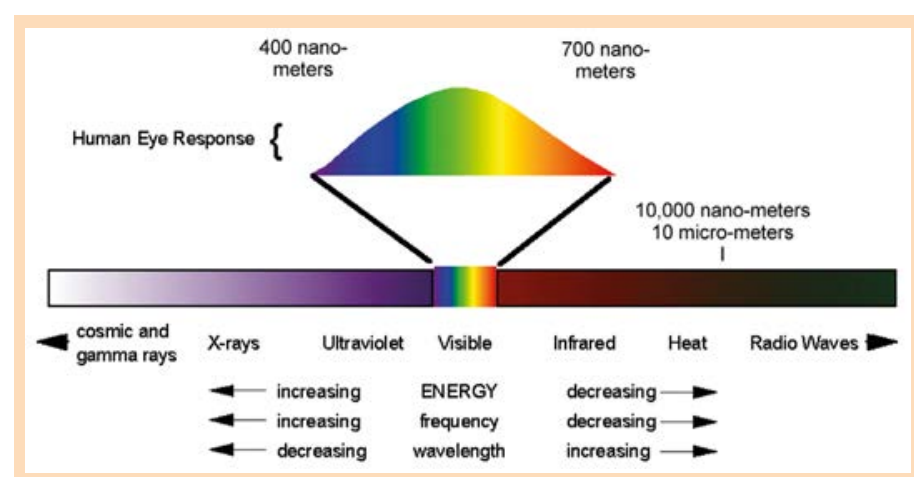

Spectre électromagnétique.

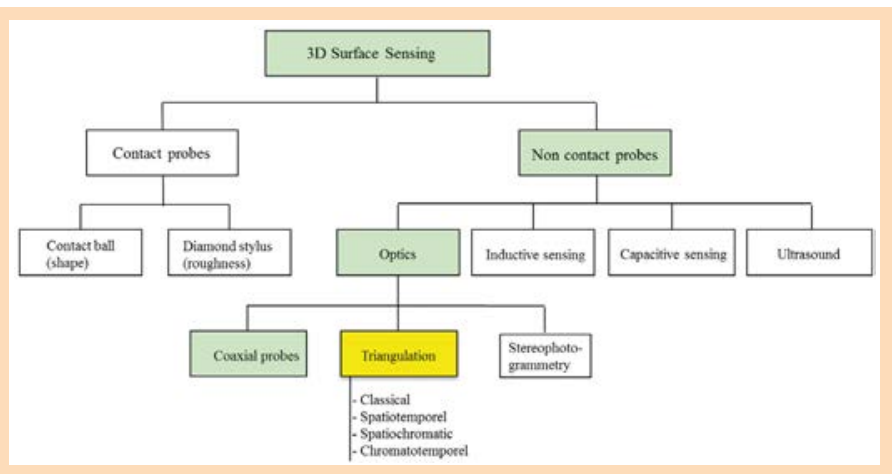

Illustration arborescence. 


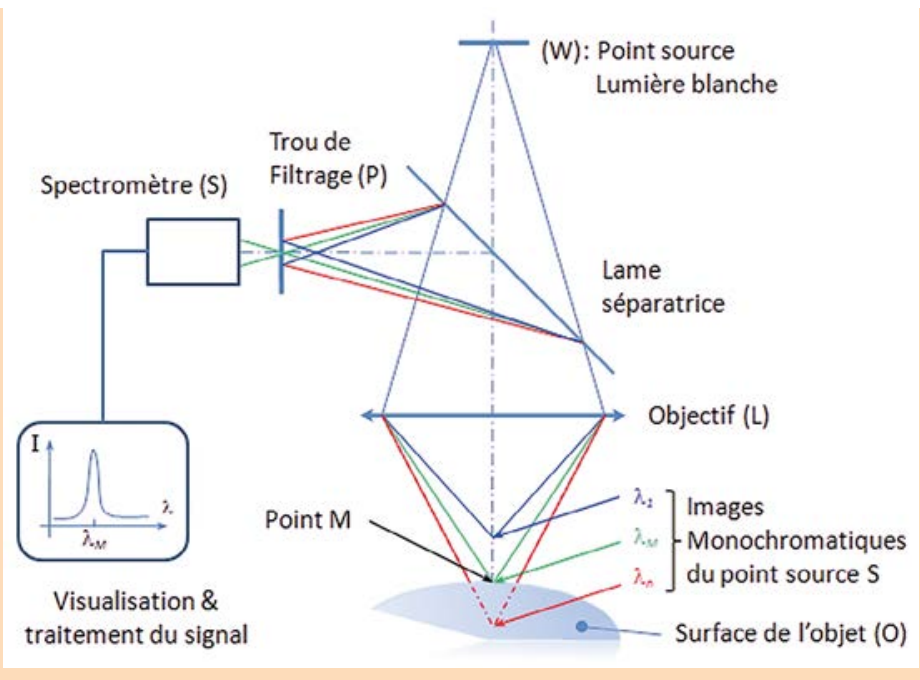

délivre un courant en conséquence et la fréquence d'excitation est adaptée en fonction de l'analyse recherchée. Cette méthode est très riche en informations, cependant l'interprétation en est difficile. Désavantage majeur, sa mise en œuvre nécessite une immersion de l'échantillon.

- Les capteurs capacitifs réalisent une mesure de tension électrique entre le capteur et la cible. Assez précise pour les mesures de distance, elle devient insuffisante pour saisir la forme des objets (effets de bord). La résolution latérale se révèle aussi insuffisante car la mesure est faite sur un niveau moyenné et non pas sur un point précis. Ces capteurs ne peuvent donc pas être utilisés pour la microtopographie ou la rugosimétrie. - Les capteurs inductifs utilisent la mesure des courants de Foucault pourdéterminer des distances, des diamètres et même des épaisseurs, malheureusement elle n'est applicable que sur des objets métalliques et magnétiques afin de pouvoir générer un champ magnétique entre le capteur et la surface de la cible. - Les capteurs optiques, dont le confocal chromatique fait partie, sont généralement classés en deux grandes familles, les capteurs coaxiaux et non coaxiaux - cette dernière se scindant en deux pour faire la distinction entre les capteurs à triangulation et la photogrammétrie :

- Les capteurs confocaux chromatiques sont dits coaxiaux dans la mesure où les faisceaux d'éclairage et d'observation utilisent principalement le même trajet optique. Cela implique que la voie d'éclairage et la voie de reprise soient confondues. - La photogrammétrie est constituée d'un minimum de deux caméras et utilise l'éclairage ambiant. Cependant, la justesse de ce système est liée à la justesse de la connaissance de la géométrie.

- Les capteurs à triangulation sont composés d'un faisceau d'éclairage et d'un faisceau de reprise non confondus. La justesse de la géométrie est compensée par ajustage.

\section{Principe confocal chromatique}

Le principe consiste à créer un faisceau lumineux polychromatique dont chaque longueurd'onde se focalise parfaitement à une position précise sur la plage de mesure. Ceci revient à créer une règle optique dont chaque couleur correspond à une distance. $\checkmark$ Figure 3. Schéma de principe du confocal chromatique.

$\checkmark$ Figure 4. Profondeur de champ chromatique. Illustration du bénéfice évident en termes de profondeur de champ de la nouvelle technologie de vision par codage chromatique (Camera Chromaline STIL).

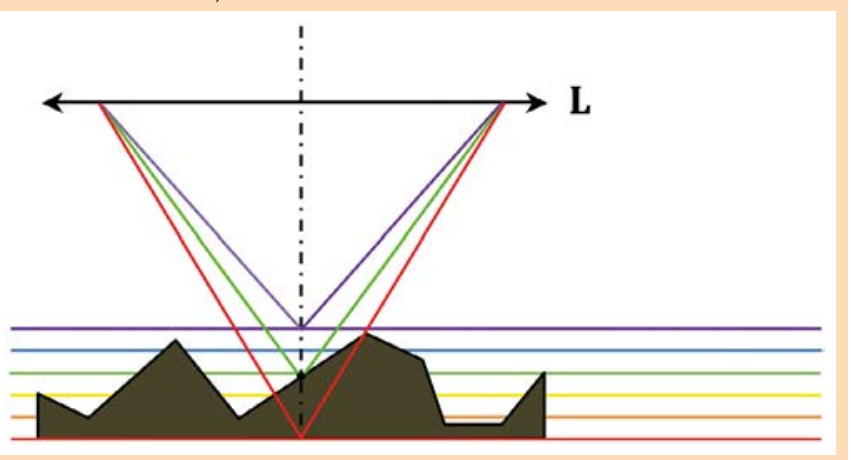

Ce faisceau coloré est projeté via un crayon optique sur la cible. La cible va renvoyer vers ce même crayon et par simple réflexion, la couleur (la longueur d'onde unique) correspondant à la position de sa surface éclairée par rapport à celle du crayon. Le crayon est associé à une électronique de traitement capable d'identifier précisément la couleur qui « touche» la cible. Il s'agit d'une mesure absolue et coaxiale, qui offre de multiples avantages par rapport aux technologies concurrentes, en particulier dans le cas des applications nécessitant une mesure de type 3D.

L'un des avantages du confocal chromatique est de répondre à une large gamme d'applications. La configuration confocale permet un rapport signal-sur-bruit très élevé et lui confere une haute résolution de mesure. La configuration coaxiale élimine les effets d'ombre et peut ainsi être utilisée pour la mesure de surfaces hautement réfléchissantes. L'encodage chromatique ainsi que le décodage ne requièrent pas de scanning en $Z$ et sont d'une très haute précision. Il existe deux modes de mesure : distance et épaisseur (pour les matériaux transparents).

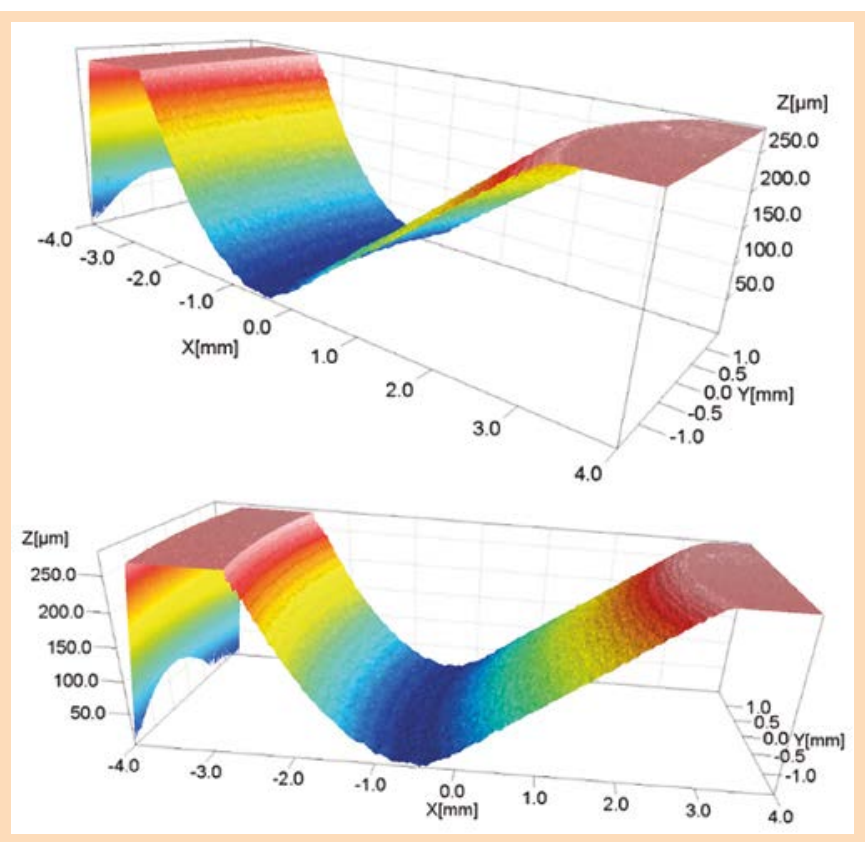

Vues 3D de la topographie du diamant polycristallin. 


\section{Capteur polyvalent et résultats multiples}

La technologie confocale chromatique, de par sa haute-résolution et sa capacité de mesure sans contact sur tout type de surface, peut ainsi être utilisée à des fins de contrôle qualité au moment de la fabrication mais aussi après utilisation d'outils de coupe afin d'en déterminer l'usure. Dans le cas de la figure 5, cette technologie optique est utilisée afin de mettre en évidence la forme et la rugosité d'un diamant polycristallin (PCD). Le PCD est un matériau fabriqué à partir de particules de diamant et d'un liant métallique. Grâce à sa solidité, le diamant est un matériau qui peut résister à l'usure par abrasion et est ainsi préconisé dans la fabrication d'outils de coupe.

La cible est placée sous le faisceau optique et la mesure est effectuée sur une surface de $24 \mathrm{~mm}^{2}$ avec une résolution latérale de 3,5 $\mu \mathrm{m}$. Cette mesure est réalisée par déplacement de la cible positionnée sur des tables de translation (axes $x$ et $y$ ). On notera que pour cette mesure le bruit statique est de l'ordre d'une dizaine de nanomètres. Les propriétés optiques du capteur sélectionné permettent de mettre en évidence à la fois la topographie de la surface courbe ainsi que sa rugosité. Après post-traitement des données brutes récoltées, l'objet présente une dépression de $265 \mu \mathrm{m}$ et un paramètre de rugosité ( $\mathrm{Ra}$ ) sur les zones utiles de $1,1 \mu \mathrm{m}$.

\section{Le présent, l'avenir}

Une grande diversité de technologies coexiste au sein de la famille des capteurs de distance sans contact, chacune offrant un avantage souvent spécifique (très haute résolution pour les capacitifs, robustesse pour les inductifs, faible coût pour les capteurs laser à triangulation). Cependant les dernières innovations apportées ces dernières années en matière de technologie chromatique (multicouche, multipoints, vision à champ étendu) permettent d'entrevoir un avenir prometteur pour une technologie qui offrait dès l'origine de significatifs avantages concurrentiels par rapport aux technologies classiques de mesure de distance sans contact. - Compatible avec la quasi-totalité des matériaux existants (verre, métal, semi-conducteur, biomatériaux, composites de toutes natures, liquides...).

- Adapté à une grande variété d'états de surface allant du parfaitement réfléchissant (miroir) au très diffusant (métal très rugueux, pièce brute de fonderie).

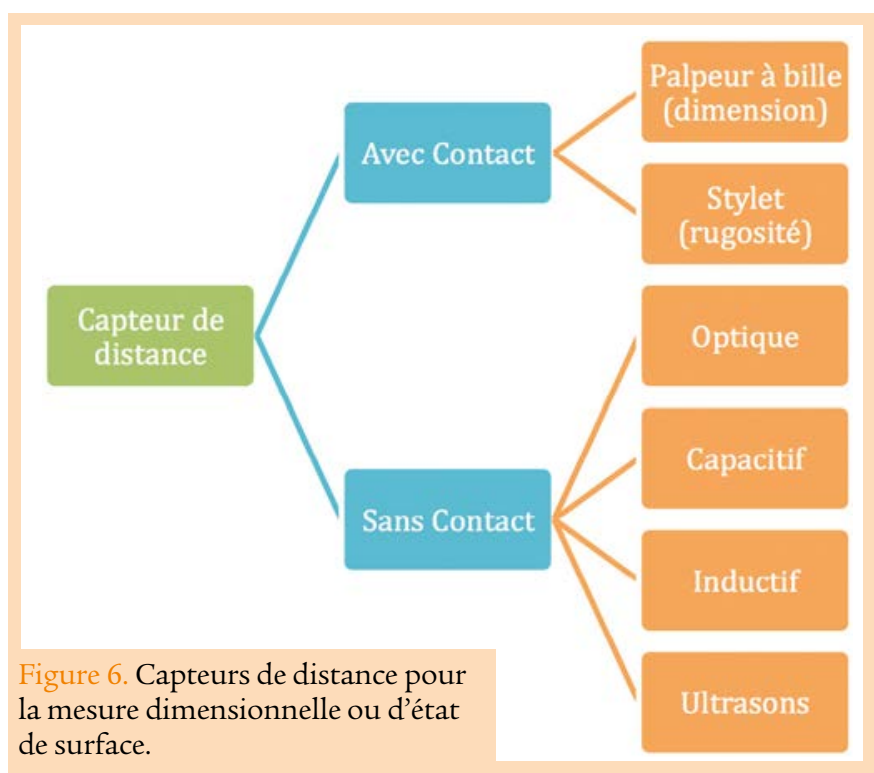

- Très résolvant et insensible aux conditions d'éclairage ambiant grâce à la taille de spot réduite et au point de focalisation spécifique à chaque longueur d'onde permettant un filtrage natif des lumières parasites extérieures.

- Capable de suivre de manière continue des surfaces complexes sans effet d'ombrage grâce à son dispositif coaxial d'émission et de captation de la lumière.

- Capteur compact et parfaitement passif, le crayon optique ne véhiculant que d es photons à basse intensité, il est utilisable en environnements confinés, explosifs voire immergés.

Ainsi les marchés et les domaines d'applications des capteurs de distance chromatique s'étendent chaque jour à de nouveaux secteurs industriels (verre, semi-conducteur, électronique, automobile, aéronautique...) et trouveront au-delà des applications de mesure dimensionnelle des débouchés dans les domaines de la métrologie des états de surface ou de l'inspection robotisée de pièces complexes.

Après les lasers, d'autres technologies optoélectroniques émergent donc et trouvent toute leur place dans les « usines du futur » pour apporter un contrôle qualité performant contribuant à de nouveaux gains de productivité.

\section{POUR EN SAVOIR PLUS}

Cet article s'appuie en partie sur la thèse de Dimitri Vaissière, «Métrologie tridimensionnelle des états de surface par microscopie confocale à champ étendu », Université Louis Pasteur, 2003.

Tableau 1. Comparatif des capteurs de distance sans contact (* Erreur de linéarité maximale).

\begin{tabular}{|l|l|l|l|l|l|}
\hline Technologie & $\begin{array}{l}\text { Étendue mesure } \\
(\mathrm{mm})\end{array}$ & $\begin{array}{l}\text { Résolution } \\
(\mathrm{nm} \text { ou \% EM) }\end{array}$ & $\begin{array}{l}\text { Précision* } \\
(\mu \mathrm{m} \text { ou \% EM) }\end{array}$ & $\begin{array}{l}\text { Fréquence } \\
\text { de mesure }\end{array}$ & Compatibilité \\
\hline Chromatique & 0,1 à $50 \mathrm{~mm}$ & $5 \mathrm{~nm}$ à $0,005 \%$ & $0,1 \mu \mathrm{m}$ à $0,05 \%$ & $20 \mathrm{kHz}$ & Toutes surfaces \\
\hline Laser & 0,5 à $1000 \mathrm{~mm}$ & $500 \mathrm{~nm}$ à $0,01 \%$ & $1 \mu \mathrm{m}$ à $0,05 \%$ & $20 \mathrm{kHz}$ & Limites sur verre \\
\hline Capacitif & 0,05 à $10 \mathrm{~mm}$ & $0,01 \%$ à $0,0001 \%$ & $0,05 \%$ à $0,025 \%$ & $50 \mathrm{kHz}$ & Limites sur verre et isolant \\
\hline Inductif & 0,5 à $100 \mathrm{~mm}$ & $0,1 \%$ à $0,005 \%$ & $0,7 \%$ à $0,2 \%$ & $100 \mathrm{kHz}$ & Métal uniquement \\
\hline
\end{tabular}

